From the National Surgical Adjuvant Breast and Bowel Project; Graduate School of Public Health, University of Pittsburgh; and Allegheny General Hospital, Pittsburgh, PA; University of Florida, Gainesville; and Florida Cancer Specialists-Sarasota, FL; Helen F. Graham Cancer Center at Christiana Care, Newark, DE; Southeast Cancer Control Consortium-Community Clinical Oncology Program (CCOP), Goldsboro, NC; Atlanta Cancer Care Regional CCOP, Atlanta, GA; Kaiser Permanente Northern California, Vallejo, CA; University of North Carolina at Chapel Hill, Chapel Hill, NC; All-Ireland Cooperative Oncology Research Group, Dublin, Ireland; and Kaiser Permanente Midtown I, Denver, CO

Submitted May 17, 2010; accepted August 25, 2010; published online ahead of print at www.jco.org on October 12, 2010.

Supported by Public Health Service Grants No. U10-CA-12027, U10-CA69651, U10-CA-37377, and U10-CA69974 from the National Cancer Institute, Department of Health and Human Services.

Authors' disclosures of potential conflicts of interest and author contributions are found at the end of this article.

Clinical Trials repository link available on JCO.org.

Corresponding author: Carmen J. Allegra, MD, University of Florida, Division of Hematology and Oncology, 1600 SW Archer Ave, Rm N-503, Gainesville, FL 32610; e-mail: Carmen.Allegra@medicine.ufl.edu. () 2010 by American Society of Clinical Oncology

$0732-183 X / 11 / 2901-11 / \$ 20.00$

DOI: $10.1200 / J C O .2010 .30 .0855$

\title{
Phase III Trial Assessing Bevacizumab in Stages II and III Carcinoma of the Colon: Results of NSABP Protocol C-08
}

Carmen J. Allegra, Greg Yothers, Michael J. O'Connell, Saima Sharif, Nicholas J. Petrelli, Linda H. Colangelo, James N. Atkins, Thomas E. Seay, Louis Fehrenbacher, Richard M. Goldberg, Seamus O'Reilly, Luis Chu, Catherine A. Azar, Samia Lopa, and Norman Wolmark

See accompanying editorial on page 1

\section{$\begin{array}{llllllll}\text { A } & \text { B } & \text { S } & \text { T } & \text { R } & \text { A } & \text { C } & \text { T }\end{array}$}

\section{Purpose}

The National Surgical Adjuvant Breast and Bowel Project C-08 trial was designed to investigate the safety and efficacy of adding bevacizumab to modified FOLFOX6 (mFOLFOX6; ie, infusional/bolus fluorouracil, leucovorin, and oxaliplatin) for the adjuvant treatment of patients with stages II to III colon cancer.

\section{Methods}

Patients received mFOLFOX6 every 2 weeks for 26 weeks alone or modified as FOLFOX6 + bevacizumab (5 mg/kg every 2 weeks for 52 weeks [ie, experimental group]). The primary end point was disease-free survival (DFS).

\section{Results}

Among 2,672 analyzed patients, demographic factors were well balanced by treatment. With a median follow-up of 35.6 months, the addition of bevacizumab to mFOLFOX6 did not result in an overall significant increase in DFS (hazard ratio [HR], 0.89; $95 \% \mathrm{Cl}, 0.76$ to $1.04 ; P=.15$ ). The point estimates for 3-year DFS for the overall population were $77.4 \%$ and $75.5 \%$ for the experimental and control arms, respectively. For patients with stages II and III diseases, these same estimates were $87.4 \%$ and $84.7 \%$, respectively, for stage $\mathrm{II}$ and $74.2 \%$ and $72.4 \%$, respectively, for stage III. Exploratory analyses found that the effect of bevacizumab on DFS was different before and after a 15-month landmark (time-by-treatment interaction $P$ value $<.0001$ ). Bevacizumab had a strong effect before the landmark $(\mathrm{HR}, 0.61 ; 95 \% \mathrm{Cl}, 0.48$ to $0.78 ; P<.001)$ but no significant effect after (HR, 1.22; $95 \% \mathrm{Cl}, 0.98$ to $1.52 ; P=.076)$.

\section{Conclusion}

Bevacizumab for 1 year with mFOLFOX6 does not significantly prolong DFS in stages II and III colon cancer. However, a significant but transient effect during bevacizumab exposure was observed in the experimental arm. We postulate that this observation reflects a biologic effect during bevacizumab exposure. Given the lack of improvement in DFS, the use of bevacizumab cannot be recommended for use in the adjuvant treatment of patients with colon cancer.

\section{J Clin Oncol 29:11-16. (C) 2010 by American Society of Clinical Oncology}

\section{INTRODUCTION}

The state-of-the-art treatment for the management of patients with stage III colon cancer is to offer 6 months of adjuvant fluorouracil (FU) -and-oxaliplatin-containing chemotherapy. Two large, multinational, randomized studies demonstrated that the addition of oxaliplatin to the combination of FU and leucovorin resulted in significant improvement in disease-free survival (DFS) when compared with FU plus leucovorin alone for the adjuvant treatment of patients with stages II and III colon cancer. ${ }^{1,2}$ The Multicenter International Study of Oxaliplatin/Fluorouracil/Leucovorin in the
Adjuvant Treatment of Colon Cancer (MOSAIC) trial compared FU, leucovorin, and oxaliplatin (FOLFOX4) with infusion/bolus FU plus leucovorin, and the National Surgical Adjuvant Breast and Bowel Project (NSABP) C-07 trial evaluated the FLOX regimen, which uses oxaliplatin plus bolus FU with leucovorin, in patients with stages II and III colon cancer. Both trials demonstrated a significant increase in DFS for patients treated with the oxaliplatin-containing regimen.

Bevacizumab, a humanized monoclonal antibody with a high binding affinity for circulating vascular endothelial growth factor A (VEGF-A), has been demonstrated to enhance the response rate, 
progression-free survival, and survival of patients with advanced colorectal cancer when added to various chemotherapeutic regimens. ${ }^{3,4}$ The cellular mechanisms of action of bevacizumab are multifactorial and include inhibition of vascular neogenesis, vascular regression, and normalization of tumor vasculature. ${ }^{5}$ Whether similar mechanisms occur and/or are relevant in micrometastatic disease in the adjuvant setting is unknown. Furthermore, the potential activity of anti-VEGF therapy for the management of patients in the adjuvant setting is unknown, because there are no published clinical trials in patients with any cancer type treated in this arena.

The primary goal of NSABP C-08 was to test the potential benefit and safety associated with the addition of bevacizumab to modified FOLFOX6 (mFOLFOX6) in the adjuvant colon cancer setting. This report summarizes the efficacy associated with the addition of bevacizumab to standard chemotherapy in the adjuvant treatment of patients with stages II and III colon cancer. The safety profile of bevacizumab in combination with chemotherapy as used in NSABP C-08 has been reported. ${ }^{6}$

\section{METHODS}

\section{Study Population}

This study was approved by institutional review committees; assurances were approved by the Department of Health and Human Services and are in accordance with the Helsinki Declaration. Written informed consent was required for participation.

Patients meeting the eligibility criteria of stages II and III colon adenocarcinoma were stratified by number of positive lymph nodes and institution, then they were randomly assigned 1:1 to receive either mFOLFOX6 for 6 months or mFOLFOX6 for 6 months plus bevacizumab for 12 months, beginning concurrently with chemotherapy. This was an openlabel study with no blinding of treatment assignment for patients, physicians, or investigators. Patients with Eastern Cooperative Oncology Group (ECOG) performance statuses of 0 or 1 were randomly assigned within a window of 21 to 50 days after surgical removal of the primary tumor, and treatment was initiated within 1 week of random assignment. Patients were excluded for any history of cerebral vascular accident; transient ischemic attack; symptomatic peripheral vascular disease; or history of an arterial thrombotic episode, such as a myocardial infarction, within the 12 months before random assignment.

\section{Regimens}

The mFOLFOX6 regimen included leucovorin $400 \mathrm{mg} / \mathrm{m}^{2}$ intravenously (IV) on day $1, \mathrm{FU} 400 \mathrm{mg} / \mathrm{m}^{2}$ IV bolus on day 1 followed by 2,400 $\mathrm{mg} / \mathrm{m}^{2}$ IV over 46 hours, and oxaliplatin $85 \mathrm{mg} / \mathrm{m}^{2}$ IV on day 1 . Bevacizumab was given on the experimental arm at a dose of $5 \mathrm{mg} / \mathrm{kg}$ IV on day 1 . All therapy was given every 2 weeks for 12 doses (ie, 6 months) or, for bevacizumab, 26 doses (ie, 1 year).

\section{Statistical Considerations}

DFS was the primary end point and was defined as colon cancer recurrence, second primary cancer of any type, or death as a result of any cause. Secondary end points included survival and toxicity related to study therapy. Random assignment was performed centrally by using a biased-coin minimization algorithm. ${ }^{7}$

Protocol C-08 was designed to have $90 \%$ power to detect a $23.4 \%$ reduction in the hazard of DFS events when 592 DFS events were observed (approximately 3 years median follow-up). The $P$ value to determine the significance of the definitive analysis was to be determined by $\alpha$ spending on the basis of the number and timing of interim analyses. Interim analyses were performed as prospectively mandated in the protocol with $148,263,386$, and 507 DFS events that used one-sided $\alpha$ levels of .00025, .0005, .001, and .001, respectively. Because the definitive analysis was performed with 603 observed
DFS events, there remained, by $\alpha$ spending, ${ }^{8}$ a one-sided $\alpha$ of .0247 for this definitive analysis. Thus, a two-sided $P$ value of not more than .049 was considered significant.

Time to an event was measured from random assignment. Other than the primary end point, all $P$ values were evaluated as significant at the twosided .05 level. Plots of time to event were prepared by the method of Kaplan and Meier. ${ }^{9}$ Hazard ratios (HRs) were calculated from Cox models, ${ }^{10}$ and $P$ values for time to event were calculated from the log-rank test. ${ }^{11}$ Proportions were compared by Fisher's exact method. ${ }^{12}$ The primary analysis was based on the intention-to-treat principal ${ }^{13}$ and excluded only patients who had no follow-up and patients not at risk for the primary end point at the time of random assignment (ie, with metastases or positive surgical margins). Smooth estimates of the underlying hazard functions were calculated by the method of Muller and Wang. ${ }^{14}$ Smooth estimates of the ratio of the underlying hazards were calculated by the method of Gilbert et al. ${ }^{15}$

\section{RESULTS}

\section{Patients}

This analysis used data collected by March 31, 2009, at which time 603 DFS events had occurred. During the 2-year period between September 2004 and October 2006, 2,710 patients were accrued onto the study: 1,356 were in the control arm, and 1,354 were in the experimental arm (Fig 1, CONSORT). A total of 18 and 20 patients in the control and experimental arms (1.5\% and $1.3 \%$, respectively) were not evaluated for efficacy because of no follow-up ( $n=15$ and 18 patients, respectively), positive surgical margins $(n=1$ patient each in control and experimental arms), or metastases at entry ( $\mathrm{n}=2$ and 1 patients, respectively). In addition, 22 patients $(1.6 \%)$ in the control arm and 15 patients in the experimental arm (1.1\%) were ineligible for other reasons but were included in the analysis. Thus, there were 1,338 and 1,334 patients in the control and experimental arms, respectively. Patient characteristics were well balanced by treatment arm (Table 1). The median follow-up was 35.6 months. Slightly more than half of the patients were younger than 60 years of age, approximately $15 \%$ were older than 70 years, and there was an equal sex distribution. Patients with stage II disease constituted approximately $25 \%$.

\section{Toxicity}

Toxicity information related to patients enrolled onto NSABP C-08 has been published recently, ${ }^{6}$ and no additional toxicity signals have been noted. The addition of bevacizumab to mFOLFOX6 was not associated with an increase in mortality, and grade 3 or greater toxicities that occurred significantly more often included hypertension, wound complications, pain, and proteinuria.

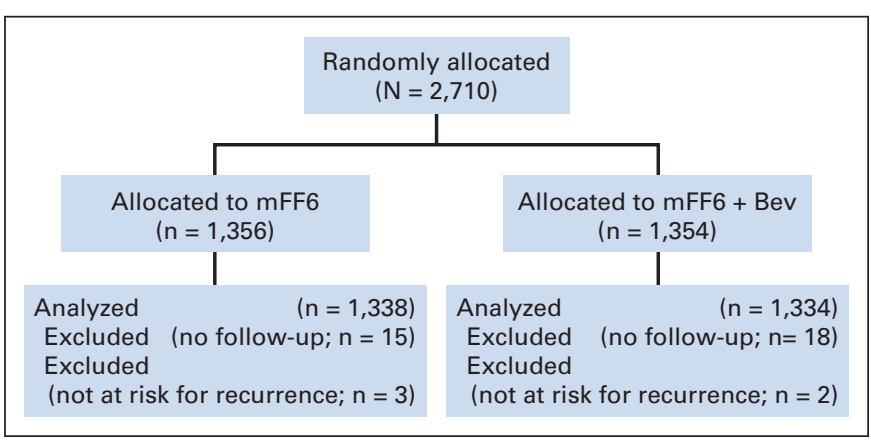

Fig 1. CONSORT diagram. Bev, bevacizumab. 


\begin{tabular}{|c|c|c|c|c|c|c|}
\hline \multirow[b]{2}{*}{ Characteristic } & \multicolumn{2}{|c|}{$\begin{array}{c}\text { mFF6 } \\
(n=1,338)\end{array}$} & \multicolumn{2}{|c|}{$\begin{array}{c}\mathrm{mFF} 6+\mathrm{Bev} \\
(\mathrm{n}=1,334)\end{array}$} & \multicolumn{2}{|c|}{$\begin{array}{c}\text { Total } \\
(\mathrm{N}=2,672)\end{array}$} \\
\hline & No. & $\%$ & No. & $\%$ & No. & $\%$ \\
\hline \multicolumn{7}{|l|}{ Age, years } \\
\hline$<50$ & 332 & 24.8 & 342 & 25.6 & 674 & 25.2 \\
\hline $50-59$ & 447 & 33.4 & 435 & 32.6 & 882 & 33.0 \\
\hline $60-69$ & 347 & 25.9 & 367 & 27.5 & 714 & 26.7 \\
\hline$\geq 70$ & 212 & 15.8 & 190 & 14.2 & 402 & 15.0 \\
\hline \multicolumn{7}{|l|}{ Sex } \\
\hline Female & 673 & 50.3 & 668 & 50.1 & 1,341 & 50.2 \\
\hline Male & 665 & 49.7 & 666 & 49.9 & 1,331 & 49.8 \\
\hline \multicolumn{7}{|l|}{ Ethnicity } \\
\hline White & 1,172 & 87.6 & 1,160 & 87.0 & 2,332 & 87.3 \\
\hline Black & 101 & 7.6 & 114 & 8.6 & 215 & 8.0 \\
\hline Other & 50 & 3.7 & 43 & 3.2 & 93 & 3.5 \\
\hline Multiracial & 2 & 0.2 & 1 & 0.1 & 3 & 0.1 \\
\hline Unknown & 13 & 1.0 & 16 & 1.2 & 29 & 1.1 \\
\hline \multicolumn{7}{|l|}{ ECOG performance } \\
\hline 0 = fully active & 1,089 & 81.4 & 1,075 & 80.6 & 2,164 & 81.0 \\
\hline $1=$ no strenuous activity & 249 & 18.6 & 259 & 19.4 & 508 & 19.0 \\
\hline \multicolumn{7}{|l|}{ Nodal stage } \\
\hline NO = node negative & 332 & 24.8 & 334 & 25.0 & 666 & 24.9 \\
\hline $\mathrm{N} 1=1-3$ positive nodes & 611 & 45.7 & 607 & 45.5 & 1,218 & 45.6 \\
\hline $\mathrm{N} 2=\geq 4$ positive nodes & 395 & 29.5 & 393 & 29.5 & 788 & 29.5 \\
\hline $\begin{array}{l}\text { Abbreviations: mFF6, modif } \\
\text { leucovorin, and oxaliplatin); B } \\
\text { Oncology Group. }\end{array}$ & , beva & izum & $\begin{array}{l}\text { infusio } \\
; \text { ECOG }\end{array}$ & $\begin{array}{l}\text { nal/bol } \\
\text { Easte }\end{array}$ & $\begin{array}{l}\text { is fluoro } \\
\text { n Coope }\end{array}$ & $\begin{array}{l}\text { uracil, } \\
\text { rative }\end{array}$ \\
\hline
\end{tabular}

\section{Treatment}

For patients randomly assigned to the control and experimental arms, $73 \%$ versus $77 \%$ were able to receive at least 10 of 12 planned doses of oxaliplatin $(P=.028)$. Similarly, $80 \%$ versus $84 \%(P=.007)$ were able to receive at least 10 of 12 doses of FU. Fifty-eight percent of patients were able to receive at least 21 of 26 planned doses of bevacizumab, and the median duration of bevacizumab administration was 11.5 months.

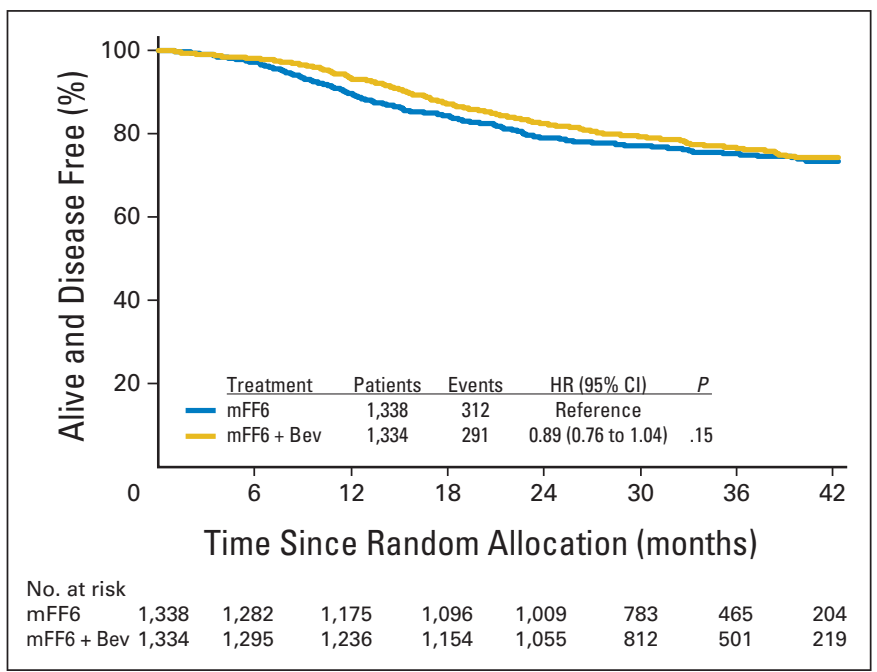

Fig 2. Disease-free survival (DFS). Overall DFS for patients treated with mFF6 (mFOLFOX6) alone for 6 months or mFF6 for 6 months plus bevacizumab for 12 months (mFF6 + Bev). HR, hazard ratio.

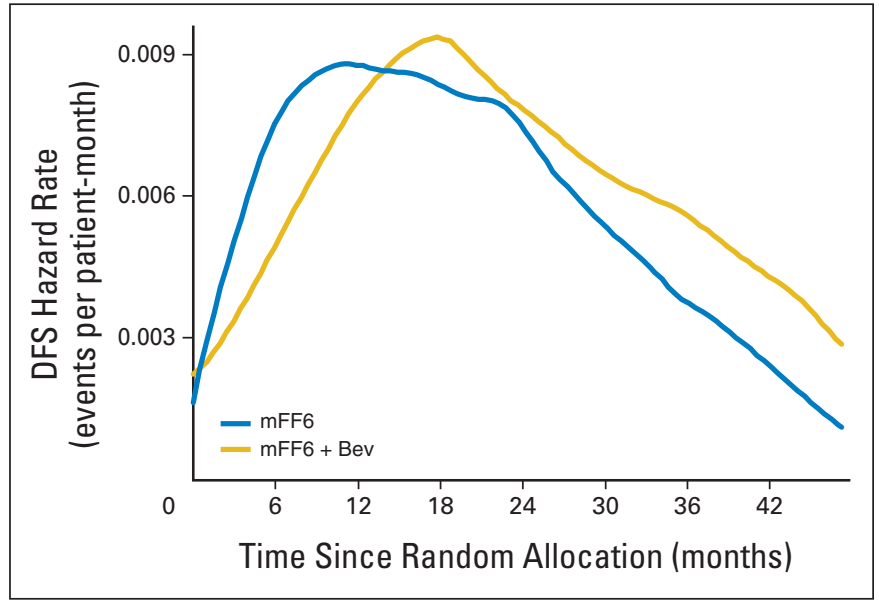

Fig 3. Disease-free survival (DFS) hazard rates. The DFS hazard rates over time are illustrated for patients treated with either mFF6 (mFOLFOX6) alone or mFF6 plus bevacizumab (mFF6 + Bev).

\section{DFS}

As shown in Figure 2, the addition of bevacizumab to mFOLFOX6 did not result in an overall significant increase in DFS (HR, 0.89; CI, 0.76 to $1.04 ; P=.15$ ). The point estimates for 3-year DFS were $77.4 \%$ and $75.5 \%$ for the experimental and control arms, respectively. The effect of bevacizumab treatment did not vary by stage (interaction $P=.68$ ). For patients with stage II disease, the 3 -year DFS rates were $87.4 \%$ and $84.7 \%$ for the experimental and control arms, respectively (HR, 0.82 ; $\mathrm{CI}, 0.54$ to $1.25 ; P=.35$ ); for patients with stage III disease, the rates were $74.2 \%$ and $72.4 \%$, respectively (HR, 0.90 ; CI, 0.76 to $1.08 ; P=.25$ ). Tests for a potential interaction of the effect of bevacizumab with sex, age, and nodal status were not statistically significant, which suggests that no group benefits more than another with the addition of bevacizumab.

Figure 3 gives the DFS hazard rates over time for each arm. These curves clearly illustrate a lower event rate in the bevacizumabcontaining arm during the first 15 months. When the HR over time is examined, as shown in Figure 4, the ratio favors the bevacizumab arm

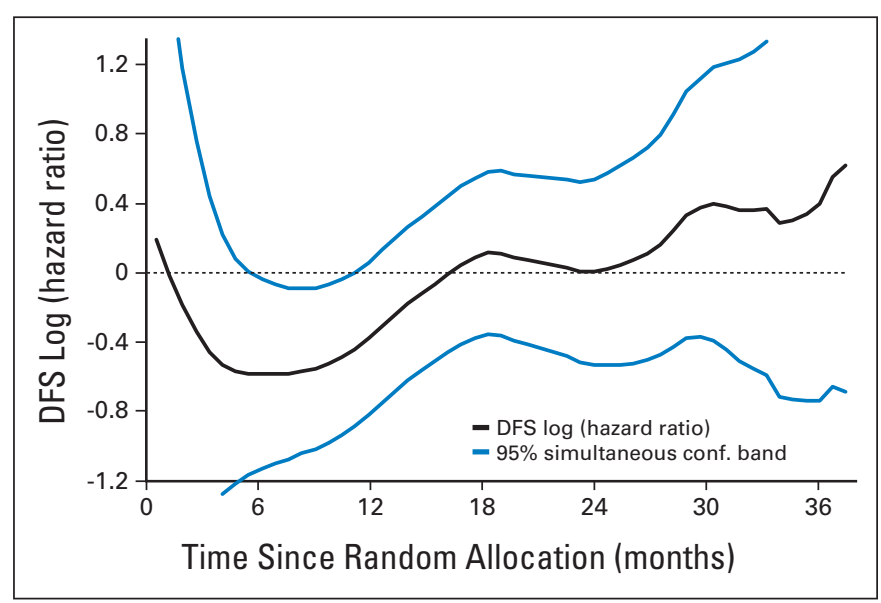

Fig 4. Disease-free survival (DFS) log (hazard ratio, HR). The smooth estimates of the logarithm of the DFS HR over time along with the $95 \%$ simultaneous confidence intervals. Values less than zero favor the bevacizumab (Bev) -containing arm. 


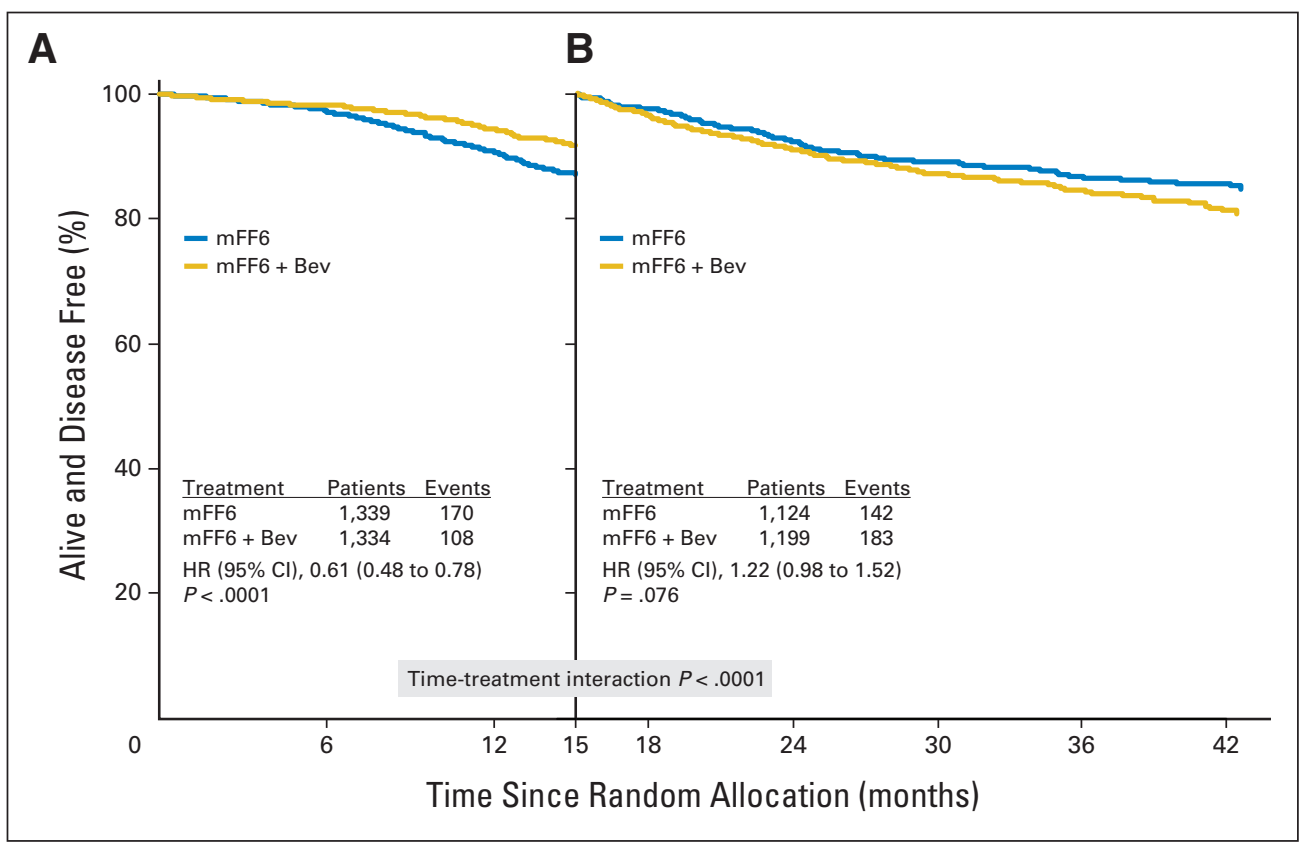

Fig 5. Disease-free survival (DFS) time by treatment interaction. (A) DFS before the 15-month landmark. The DFS during this initial period strongly and significantly favored the bevacizumab (Bev) arm (hazard ratio [HR], 0.61). (B) DFS subsequent to the 15-month landmark. The difference between the curves during this time was not significant (HR, 1.22). The time-bytreatment interaction using the 15-month landmark is highly significant $(P<.0001)$.

during therapy and at up to 3 months after completion of bevacizumab. Importantly, the simultaneous confidence band for the log of the HR excludes zero (no difference) from 5 to 11.5 months, which indicates a statistically significant improvement in DFS for bevacizumab during this interval.

The apparent time-varying treatment effect observed in Figures 2 through 4 prompted an exploratory analysis to examine this apparent initial effect of bevacizumab. We used the 15-month landmark to dichotomize time, because the maximum separation of the DFS Kaplan-Meier estimates is near 15 months (Fig 2); the hazard rate estimates cross near 15 months (Fig 3); and the log HR estimate crosses zero near 15 months (Fig 4). We identified a highly significant interaction between time and treatment on DFS $(P<.0001)$, which suggests the effect of bevacizumab on DFS is different before the 15-month landmark (ie, period of bevacizumab therapy plus washout time) than after the landmark. The HR before the landmark strongly favored bevacizumab ( HR, 0.61 ; CI 0.48 to 0.78 ; $P<.001$ ), whereas this benefit was entirely lost subsequently (HR, 1.22; CI, 0.98 to $1.52 ; P=.076$; Figs $5 \mathrm{~A}$ and $5 \mathrm{~B}$ ).

\section{Potential Rebound Effect}

Two hundred forty-eight patients experienced recurrence in the control arm versus 227 in the experimental arm; 146 died in the control arm versus 132 in the experimental arm; and 46 in the control arm versus 47 in the experimental arm had a second malignancy. None of these outcomes differed significantly between the arms. To additionally evaluate the possibility that anti-VEGF exposure resulted in the development of a more aggressive phenotype, as suggested by several preclinical studies, ${ }^{16-20}$ we compared survival after recurrence for patients with recurrent disease. We found that $41 \%$ of patients in the control arm and $37 \%$ of patients in the experimental arm were alive 2 years after recurrence; this difference was not statistically significant. The proportion of patients with more than a single site of recurrence and the organ distribution of recurrence were also similar between the arms.

\section{Imaging Follow-Up}

Because differences in the timing of follow-up imaging could impact the observed differences in DFS, we investigated the timing of imaging in each arm of the study. Because the timing of imaging studies was not specified in the study protocol, we retrospectively collected the date of first diagnostic imaging for 2,219 (81.9\%) of 2,710 patients; 1,795 of these patients did not have a recurrence or second primary cancer diagnosed during the first 2.5 years on study and thus are useful for estimating time to first imaging. These 1,795 patients have similar patient characteristics to the corresponding subset of the overall trial.

The overall time to imaging did not differ significantly between the two arms by the log-rank test $(P=.15)$, and an HR from a Cox model did not exclude 1.0 (HR, 0.93; CI, 0.83 to 1.03; Appendix Fig $\mathrm{A} 1$, online only). However, the median time to first imaging was 10.75 months (CI, 10.06 to 11.54 months) in the control arm, and 12.03 months (CI, 11.64 to 12.43 months) in the experimental arm-a significant difference of 1.28 months $(P<.05)$. Many of the $52 \%$ of patients with first imaging in the interval of 6 to 22 months may have had delayed imaging in the experimental arm. The difference in areas under the Kaplan-Meier curves (shaded region) gives an estimate of 1.09 months for the average delay in imaging in the experimental arm for these patients and, therefore, serves as an estimate of the possible bias introduced into the DFS end point by differential timing of imaging. Although some proportion of patients would have been diagnosed in an unbiased fashion, we assume all patients in the experimental arm with recurrence or second primary cancer have event times biased by 1.09 months, an assumption that may overcorrect for the true bias.

Appendix Figure A2 (online only) presents the overall biasadjusted results for DFS. The HR (0.90; CI, 0.77 to 1.06$)$ and $P$ value (.19) are similar to the unadjusted results given in Figure 2. The time-by-treatment interaction on DFS at the 15-month landmark, illustrated in Appendix Figures A3A and A3B (online only), remains significant after bias adjustment $(P=.0066)$, as does the result before the landmark strongly favoring bevacizumab $(\mathrm{HR}, 0.71 ; \mathrm{CI}, 0.56$ to $0.89 ; P=.0033)$. 


\section{DISCUSSION}

The NSABP C-08 study represents the first prospective, randomized investigation of the use of anti-VEGF therapy for the adjuvant treatment of any malignancy. Unfortunately, the addition of 1 year of bevacizumab to 6 months of modified FOLFOX6 chemotherapy in patients with stages II and III colon cancer did not significantly improve DFS. However, DFS appeared to be significantly improved with the addition of bevacizumab to modified FOLFOX6 during the 15-month period of bevacizumab exposure. This effect on DFS diminished over the subsequent follow-up, such that DFS was not significantly different between the two arms. The initial favorable DFS for the bevacizumab-treated group is supported by the hazard rates and ratios illustrated in Figures 3 and 4. The hazard rates shown in Figure 3 demonstrate an initial lower event rate in the bevacizumab arm followed by a rate that is equal to or greater than the rate observed in the control arm. This change in rate after bevacizumab exposure accounts for the loss of an initial favorable DFS associated with bevacizumab. Of interest is that the 3-year DFS for patients with stage III disease noted in this study with use of the mFOLFOX6 regimen is indistinguishable from the oxaliplatin arms of NSABP C-07 that used the FLOX regimen and the MOSAIC trial that used FOLFOX4. ${ }^{21}$

The effect on DFS associated with bevacizumab during exposure to this agent may be explained by several possible mechanisms. The observation may reflect a purely cytostatic effect that results from bevacizumab exposure with gradual abatement after therapy. This concept is consistent with the known mechanisms of action of bevacizumab and suggests that exposure results in growth arrest rather than cell death. ${ }^{5}$ It is also plausible that bevacizumab is masking the early appearance of recurrent disease by diminishing the excessive permeability associated with tumor vasculature detected by imaging studies. Whether the DFS curves will continue to merge over time or will remain modestly separated will be critical in determining whether there is any evidence for a cytotoxic effect associated with the use of bevacizumab in this adjuvant setting.

An alternative explanation for the observed DFS difference relates to the potential for bias in the timing of routine follow-up imaging, given that there was a 6-month difference in duration of therapy between the treatment arms and routine imaging after therapy, which was not prescribed by the protocol. Of patients who did not reach a DFS event during the study follow-up, we observed a statistical delay in the median time to imaging of 1.28 months in the bevacizumab arm compared with the control arm and an average delay of 1.09 months during the period between 6 and 22 months, during which the greatest potential for imaging bias appeared to occur. The bias-adjusted DFS curves resulted in little change from the unadjusted DFS curves. The DFS-by-time interaction remained significant, and the HR of the adjusted curves was 0.90 compared with 0.89 in the unadjusted DFS curves. Thus, the timing of scans after therapy completion does not explain the time-varying effect of bevacizumab on DFS.

Several recent publications using preclinical murine models have suggested that exposure to anti-VEGF therapy may result in the development of a more aggressive tumor phenotype with a greater propensity for growth and metastatic spread. ${ }^{16-20}$ Such an effect could account for an initial favorable impact of bevacizumab followed by its loss as a result of the evolution of a more aggressive cancer in the bevacizumab-exposed patients. Our investigation of patient survival after relapse as well as the organ distribution and number of metastatic sites at relapse revealed no differences between the groups. Thus, the development of a more aggressive cancer phenotype associated with bevacizumab exposure was not supported by our data. Although there are several possible explanations for the observed effect of bevacizumab on DFS, it appears most plausible that the observed transient effect on DFS associated with bevacizumab exposure represents a biologic effect.

One of the critical issues in this study design was the selection of 1 year of bevacizumab therapy, with 6 months of bevacizumab given as a single agent. The available data from the advanced disease colorectal setting suggests that bevacizumab has little discernable clinical benefit when used as a single agent. However, there is no data concerning the use of bevacizumab in the adjuvant treatment of any cancer either in combination with chemotherapy or as a single agent. Given this lack of relevant data coupled with its unknown mechanism of action in the adjuvant setting, we elected to use a 1-year total exposure on the basis of the NSABP clinical experience with trastuzumab therapy in the adjuvant breast setting. In breast cancer, the biologic therapy has direct cellular effects on the malignant cell population and thus represents an imperfect, but potentially useful, model. If other colon cancer adjuvant studies, such as the Phase III Randomized Study of Adjuvant Oxaliplatin, Leucovorin Calcium, and Fluorouracil (FOLFOX-4) Versus Bevacizumab and FOLFOX-4 Versus Bevacizumab, Oxaliplatin, and Capecitabine in Patients With High-Risk Stage II or Stage III Colon Cancer (AVANT) clinical trial, ${ }^{22}$ confirm our observation of significant suppression of tumor recurrence during bevacizumab exposure only, this would provide supporting evidence of a biologic effect of bevacizumab.

We did not observe a statistically significant benefit in overall DFS with the use of bevacizumab for 1 year. Therefore, this agent should not be used for the management of patients with colon cancer in the adjuvant setting at this time. However, we did find evidence to support a transient biologic effect of bevacizumab that will require verification from other ongoing and planned clinical investigations.

On the basis of the results of this investigation, which showed a lack of benefit associated with the use of bevacizumab for 1 year, bevacizumab should not be used for the management of patients with stages II and III colon cancer in the adjuvant setting.

\section{AUTHORS' DISCLOSURES OF POTENTIAL CONFLICTS} OF INTEREST

Although all authors completed the disclosure declaration, the following author(s) indicated a financial or other interest that is relevant to the subject matter under consideration in this article. Certain relationships marked with a "U" are those for which no compensation was received; those relationships marked with a "C" were compensated. For a detailed description of the disclosure categories, or for more information about ASCO's conflict of interest policy, please refer to the Author Disclosure Declaration and the Disclosures of Potential Conflicts of Interest section in Information for Contributors.

Employment or Leadership Position: None Consultant or Advisory Role: Carmen J. Allegra, Genentech (C); Greg Yothers, Genentech (U); Michael J. O'Connell, Genentech (C), sanofi-aventis (C); Richard M. Goldberg, Genentech (C), sanofi-aventis (C); Norman Wolmark, Genentech (U) Stock Ownership: None Honoraria: Carmen J. Allegra, Genentech, sanofi-aventis; Seamus O'Reilly, Amgen, sanofi-aventis Research Funding: Louis Fehrenbacher, Genentech Studies; Richard M. 
Goldberg, Genentech, sanofi-aventis; Seamus O’Reilly, Amgen; Luis Chu, Genentech; Norman Wolmark, Genentech Expert Testimony: None Other Remuneration: None

\section{AUTHOR CONTRIBUTIONS}

Conception and design: Carmen J. Allegra, Greg Yothers, Michael J. O'Connell, Louis Fehrenbacher Administrative support: Carmen J. Allegra, Greg Yothers, Saima Sharif
Provision of study materials or patients: Carmen J. Allegra, James N. Atkins, Louis Fehrenbacher, Richard M. Goldberg, Seamus O’Reilly, Luis Chu, Catherine A. Azar

Collection and assembly of data: Carmen J. Allegra, Greg Yothers,

Saima Sharif, Thomas E. Seay, Luis Chu, Catherine A. Azar

Data analysis and interpretation: Carmen J. Allegra, Greg Yothers, Michael J. O'Connell, Saima Sharif, Linda H. Colangelo, Louis Fehrenbacher, Richard M. Goldberg, Samia Lopa, Norman Wolmark Manuscript writing: All authors Final approval of manuscript: All authors

\section{REFERENGES}

1. André $T$, Boni $C$, Mounedji-Boudiaf $L$, et al: Oxaliplatin, fluorouracil, and leucovorin as adjuvant treatment for colon cancer. N Engl J Med 350:23432351, 2004

2. Kuebler JP, Wieand HS, O'Connell MJ, et al: Oxaliplatin combined with weekly bolus fluorouracil and leucovorin as surgical adjuvant chemotherapy for stage II and III colon cancer: Results from NSABP C-07. J Clin Oncol 25:2198-2204, 2007

3. Hurwitz H, Fehrenbacher L, Novotny W, et al: Bevacizumab plus irinotecan, fluorouracil, and leucovorin for metastatic colorectal cancer. N Engl J Med 350:2335-2342, 2004

4. Saltz LB, Clarke S, Díaz-Rubio E, et al: Bevacizumab in combination with oxaliplatin-based chemotherapy as first-line therapy in metastatic colorectal cancer: A randomized phase III study. J Clin Oncol 2013-2019, 2008

5. Ellis LM, Hicklin DJ: VEGF-targeted therapy: Mechanisms of anti-tumour activity. Nat Rev Cancer 8:579-591, 2008

6. Allegra $\mathrm{CJ}$, Yothers $\mathrm{G}, \mathrm{O}^{\prime}$ Connell $\mathrm{MJ}$, et al: Initial safety report of NSABP C-08: A randomized phase III study of modified FOLFOX6 with or without bevacizumab for the adjuvant treatment of pa- tients with stage II or III colon cancer. J Clin Oncol 27:3385-3390, 2009

7. White SJ, Freedman LS: Allocation of patients to treatment groups in a controlled clinical study. Br J Cancer 37:849-857, 1978

8. Jennison C, Turnbull BW: Flexible Monitoring: The error spending approach, in Jennison C, Turnbull BW (eds): Group Sequential Methods with Applications to Clinical Trials. Boca Raton, LA, Chapman and Hall/CRC, 2000

9. Kaplan EL, Meier P: Nonparametric estimation from incomplete observations. J Am Stat Assoc 53:457-481, 1958

10. Cox DR: Regression Models and Life Tables. J Royal Stat Soc Series B 34:187-220, 1972

11. Mantel N: Evaluation of survival data and two new rank order statistics arising in its consideration. Cancer Chemother Rep 50:163-170, 1966

12. Fisher RA: On the interpretation of $\chi^{2}$ from contingency tables, and the calculation of P. J Royal Stat Soc B 85:87-94, 1922

13. Lachin JM: Statistical considerations in the intent-to-treat principle. Control Clin Trials 21:167189, 2000

14. Müller HG, Wang JL: Hazard rate estimation under random censoring with varying kernels and bandwidths. Biometrics 50:61-76, 1994

15. Gilbert PB, Wei LJ, Kosorok MR, et al: Simultaneous inferences on the contrast of two hazard functions with censored observations. Biometrics 58:773-780, 2002

16. Ebos JM, Lee CR, Cruz-Munoz W, et al: Accelerated metastasis after short-term treatment with a potent inhibitor of tumor angiogenesis. Cancer Cell 15:232-239, 2009

17. Pàez-Ribes $M$, Allen $E$, Hudock J, et al: Antiangiogenic therapy elicits malignant progression of tumors to increased local invasion and distant metastasis. Cancer Cell 15:220-231, 2009

18. Loges $S$, Mazzone $M$, Hohensinner $P$, et al: Silencing or fueling metastasis with VEGF inhibitors: Antiangiogenesis revisited. Cancer Cell 15:167-170, 2009

19. Reynolds AR, Hart IR, Watson AR, et al: Stimulation of tumor growth and angiogenesis by low concentrations of RGD-mimetic integrin inhibitors. Nat Med 15:392-400, 2009

20. Ellis LM, Reardon DA: Cancer: The nuances of therapy. Nature 458:290-292, 2009

21. Sharif $S, O^{\prime}$ Connell MJ, Yothers $G$, et al: FOLFOX and FLOX regimens for the adjuvant treatment of resected stage II and III colon cancer. Cancer Invest 26:956-963, 2008

22. de Gramont A, Tournigand $C$, André T, et al: Targeted agents for adjuvant therapy of colon cancer. Semin Oncol 33:S42-S45, 2006 (suppl 11) 